\title{
Intracytoplasmic Sperm Injection after Vitrification of Immature Oocytes in Follicular Fluid Increases Bovine Embryo Production
}

\author{
Joana Claudia Mezzalira', Lain Uriel Ohlweiler', Norton Klein², \\ Daniela dos Santos Brum², Fábio Gallas Leivas² \& Alceu Mezzalira'
}

\begin{abstract}
Background: Despite the low efficiency caused by its harmful effects, vitrification is the technique of choice for oocyte cryopeservation, especially at the germinal vesicle (GV) stage. This enables the banking of female gametes without linkage to the male genotype. Follicular fluid (FF), in vivo, is known to provide an adequate environment to the immature oocyte. The intra-cytoplasmic sperm injection (ICSI), by the other hand, can be used to bypass any sperm penetration disorder, including the ones caused by cryopreservation. This study aimed to evaluate oocyte vitrification in FF based solution, and to asses ICSI efficiency in the fertilization of vitrified/warmed bovine GV oocytes.

Material, Methods \& Results: Follicles of 2-8 $\mathrm{mm}$ in diameter were aspirated from bovine ovaries obtained from a slaughterhouse, selected and maintained into FF from aspiration, until their allocation in the experimental groups. The FF used to prepare the vitrification solution was centrifuged, heat inactivated, filtered through a $0.22 \mathrm{~mm}$ pore and stored at $-20^{\circ} \mathrm{C}$. Oocyte vitrification was done into one of these three solutions: The standard solution TCM-Hepes (TH-Vitri) was compared to a totally FF based solution (FF-Vitri), and to a 50:50 (v/v) mix of both solutions (TH:FF-Vitri). Oocytes were submitted to in vitro embryo production in order to assess embryo production efficiency. A second set of experiments using the FF-Vitri solution compared IVF versus ICSI. With basis on cleaved structures, the morula + blastocyst rate obtained in the Fresh Control (43.9\%) was similar to FF-Vitri (31.1\%). Conversely, the TH-Vitri (15.7\%) and the TH:FF-Vitri (20.4\%) rates were significantly lower than the Fresh Control. ICSI showed a positive effect in comparison with IVF. The embryo development rate of Vitri-IVF (18.8\%) was the lowest, whereas Vitri-ICSI (37.3\%) was similar to the Fresh-IVF (43.9\%), but lower than the Fresh-ICSI (57.8\%).

Discussion: Oocytes cryopreserved in TH based solution are known to show certain rigidity in the zona pellucida, being this event a possible cause to spermatozoa penetration disruption. Our results agree with that, since the fertilization rate for TH-Vitri was significantly lower than for the FF-Vitri. In contrast, GV oocytes vitrified in total versus partial FF based solution showed similar maturation and fertilization rates as the Fresh Control, evidencing the beneficial effect of FF during the course of vitrification. It is possible that FF helped to adjust oocyte maturation, allowing a better nuclear-cytoplasmic synchrony. Also, it might have provided some protection due to its antioxidant properties. The releasing of cortical granules induced by freezing, lead to a zona pellucida hardening and failure in sperm penetration. Factors present in the FF might block this premature releasing of cortical granules, thus ensuring that the egg retains its ability to be fertilized after maturation. The blastocysts produced from the FF-Vitri oocytes were the only ones that had the average ICM similar to the Fresh Control, evidencing that besides the similarity in morula + blastocyst rates, the embryos derived from oocytes vitrified in FF solution have also yielded best quality. When vitrified warmed oocytes were submitted to ICSI, there was an increase in the blastocyst production. This increment of embryo production with ICSI evidences a pathway to overcome the zona pellucida biological barrier. In conclusion, the use of FF as base for vitrification solution improves further embryo development; ICSI increases the embryo production of vitrified/warmed bovine GV stage oocytes.
\end{abstract}

Keywords: oocyte cryopreservation, GV oocytes, ICSI, IVF, IVP, ART. 


\section{INTRODUCTION}

Vitrification is the technique of choice for cryopreserving oocytes, but further embryo development remain still poor, as a low percentage of oocytes remains competent to undergo normal development [11]. Results are still inconsistent due to harmful effects to the nucleus [13], to the zona pellucida [5], or to injuries in the cytoplasm.

Follicular fluid (FF), who has the ability to inhibit germinal vesicle breakdown of bovine oocytes [1] had some defined components already described, which are known to play important roles until the completion of fertilization. Such roles include inhibiting zona pellucida modifications $[9,10]$, enhancing cytoplasmic maturation, and providing important promoting factors [21]. For the reasons stated here, FF makes a promising substance to be used in the course of GV stage oocyte vitrification.

The intra-cytoplasmatic sperm injection (ICSI), by the other hand, appears as a suitable tool to overcome the negative effects of oocyte cryopreservation. Indeed, previous reports showed ICSI to be successfully employed to increase embryo production rates of oocytes presenting an impaired quality $[15,18]$.

This study aimed to evaluate the developmental efficiency and quality of embryos derived from $\mathrm{GV}$ oocytes vitrified in a base solution partially or totally composed of FF; and to compare the embryo production and quality after fertilizing oocytes using conventional in vitro fertilization (IVF) or ICSI, after their vitrification at the GV stage in FF based solution.

\section{MATERIALS AND METHODS}

All chemicals were purchased from SigmaAldrich ${ }^{1}$.

Oocyte collection

Bovine ovaries obtained from a slaughterhouse had their follicles measuring 2-8 mm of diameter aspirated. Recovered cumulus-oocyte complexes (COCs) were maintained into FF throughout classification, being used only the ones classified as quality grade I or II [4]. Oocytes were maintained in FF (within up to $6 \mathrm{~h}$ after slaughter), until they were randomly allocated to the experimental groups.

\section{Preparation of FF and vitrification base solutions}

The FF used for vitrification was obtained as described for the oocyte collection, centrifuged at $5000 \mathrm{x} g$ for $20 \mathrm{~min}$, inactivated by heat at $45^{\circ} \mathrm{C}$ during $35 \mathrm{~min}$, and filtered through a $0.22 \mathrm{~mm}$ pore, then stored at $-20^{\circ} \mathrm{C}$, until its use.

The three different vitrification solutions were: standard Hepes-buffered TCM-199 + 20\% of estrous mare serum (TH-Vitri) solution, total FF base solution (FF-Vitri), and 50:50\% (v/v) TH and FF base (TH:FFVitri) solution.

Before mixing with cryoprotectants, each solution was stabilized at $7^{\circ} \mathrm{C}$ for $30 \mathrm{~min}$ to avoid precipitation of FF proteins during mixing.

\section{Experimental design}

The first experiment (four replications) compared GV oocyte vitrification in a base solution totally (FF), partially (TH:FF), or not composed $(\mathrm{TH}=$ standard solution) of follicular fluid. The FF based solution showed the best result, so the second experiment (five replications) compared IVF versus ICSI to fertilize GV stage oocytes previously vitrified in FF based solution (fresh oocytes were also fertilized to be used as controls). Viability criteria were based on: maturation and fertilization rates at D1 (first experiment); and in the rates of cleavage at D2, morula and blastocyst at D7, and in the blastocyst quality (first and the second experiments).

\section{Oocyte vitrification and warming}

Oocytes to be vitrified in TH based solutions (TH or TH:FF) were exposed to them for 8 to $10 \mathrm{~min}$ prior to the onset of vitrification steps, being the mix with cryoprotectants performed on a cooled $\left(7^{\circ} \mathrm{C}\right)$ surface. The vitrification dish was then warmed at $38.5^{\circ} \mathrm{C}$ for $30 \mathrm{~min}$. The vitrification steps consisted in the exposure of 3 to 5 oocytes to the base solution containing $10 \%$ of dimethylsulfoxide (DMSO) and $10 \%$ of ethylene glycol (EG), for $30 \mathrm{~s}$ and then to $20 \%$ DMSO and 20\% EG. Oocytes were aspirated (by capillarity) to the tip-end of a glass pipette, and immediately plunged into liquid nitrogen in the time lapse of 20 to $25 \mathrm{~s}$. The vitrified oocytes were maintained for at least $1 \mathrm{~h}$ into the liquid nitrogen $\left(\mathrm{LN}_{2}\right)$, before proceeding to the 2-step warming. To that, the glass pipette was removed from the $\mathrm{LN}^{2}$ and immersed into $\mathrm{TH}$ solution added of $0.4 \mathrm{M}$ of sucrose (first step) at $38.5^{\circ} \mathrm{C}$, for $5 \mathrm{~min}$, being the released oocytes transferred to $\mathrm{TH}$ added of $0.2 \mathrm{M}$ sucrose (second step) for additional 5 min. After warming, oocytes were maintained into $\mathrm{TH}$ solution for $10 \mathrm{~min}$, and finally transferred to the in 
vitro maturation (IVM) dish. A group of oocytes was maintained as a Fresh Control.

\section{IVM procedure and incubation conditions}

Oocytes were maturated in groups of 30-40, into 4-well dishes containing $400 \mu \mathrm{L}$ of modified tissue culture medium, added of $5.95 \mathrm{mg} / \mathrm{mL}$ of HEPES, $2.62 \mathrm{mg} / \mathrm{mL}$ of $\mathrm{NaHCO}_{3}, 0.025 \mathrm{mg} / \mathrm{mL}$ of sodium pyruvate, $0.01 \mathrm{IU} / \mathrm{mL}$ of FSH, $0.5 \mu \mathrm{g} / \mathrm{mL}$ of $\mathrm{LH}$ and $10 \%$ of estrus mare serum (EMS). The maturation was performed at $38.5^{\circ} \mathrm{C}$ and $5 \% \mathrm{CO}_{2}$ in air and saturated humidity, for 20 to $24 \mathrm{~h}$.

\section{Semen preparation and in vitro fertilization (IVF)}

A sample of frozen-thawed semen from a Bos taurus bull was thawed in water bath at $37^{\circ} \mathrm{C}$ for $20 \mathrm{~s}$ and selected by mini-Percoll [12] gradients of 90 and $45 \%$. Selected sperm cells were adjusted to a concentration of $1.5 \times 10^{6}$ spermatozoa $/ \mathrm{mL}$. Then they were co-incubated with the oocytes during 18-20 h, into 4-well dishes containing $400 \mu \mathrm{L}$ of Talp-Fert [16] medium added of $6 \mathrm{mg} / \mathrm{mL}$ of bovine serum albumin (BSA), $0.022 \mathrm{mg} / \mathrm{mL}$ of sodium pyruvate, $30 \mu \mathrm{g} / \mathrm{mL}$ of heparin and PHE (penicillamine, hypotaurine and epinephrine). The incubation conditions were the same as described for IVM.

\section{Assessment of nuclear status of presumptive zygotes}

After IVF, cumulus cells were removed from presumptive zygotes by successive pipetting in $\mathrm{TH}$, being then fixed in absolute ethanol with $10 \mu \mathrm{g} / \mathrm{mL}$ bisbenzimide (Hoechst 33342) and placed in a glycerol drop between glass slide and coverslip. The evaluation was performed through epi-fluorescent microscope ${ }^{2}$ to determine the nuclear developmental status, as previously described [20], being classified as: 1- maturated oocytes; 2- penetrated oocytes. Penetrated oocytes were subclassified in either: 2.1- polyspermic zygotes, or: 2.2- fragmented zygotes.

\section{Intracytoplasmic sperm injection (ICSI)}

ICSI and oocyte activation procedures were performed as previously described [16], using a micromanipulation station (Narishige IM-9B) ${ }^{3}$ set up with an inverted microscope (Olympus IX51) ${ }^{2}$. After IVM oocytes had their cumulus cells removed by successive pipetting in $\mathrm{TH}$, selected upon the presence of the first polar body and placed into $25 \mu \mathrm{L}$ droplets of Hepesbuffered TCM-199 added of $6 \mathrm{mg} / \mathrm{mL}$ of BSA, under mineral oil, in groups of 5 MII oocytes.
Previously selected (by mini-Percoll) spermatozoa were maintained into a droplet $(15 \mu \mathrm{L})$ of PVP-40 (10\% polyvinylpyrrolidone, in HEPES-buffered TCM199). A motile sperm was aspirated into the injection micropipette; the ooplasm of the oocyte (attached to a holding micropipette) was strongly aspirated until its oolema was broken down to allow the sperm to be injected into the oocyte cytoplasm. Oocytes were activated by $5 \mathrm{~min}$ exposure to $5 \mu \mathrm{M}$ of ionomycin and 2.5 - $3 \mathrm{~h}$ incubation in $2 \mathrm{mM}$ of 6-Dimethyl aminopurine. The incubation conditions were the same as described for IVM and IVF. Activated oocytes were then placed in the in vitro culture (IVC) dish.

In vitro culture (IVC)

Embryos were cultured into 4-well dishes containing $400 \mu \mathrm{L}$ of SOFaaci medium [8] added of 5\% EMS under mineral oil, as previously described [15]. The first 2 days of culture were performed at $38.5^{\circ} \mathrm{C}$ with $5 \% \mathrm{CO}_{2}$ in air and saturated humidity. From day two to day seven, the IVC dish was sealed into a foil bag, filled with lung air, blown (always from the same operator) after $4 \mathrm{~s}$ of apnea. The lung air provided a $5 \%$ $\mathrm{CO}_{2}$ atmosphere (data not shown), and was replaced on day five.

Total Cell Number (TCN) and Inner Cell Mass (ICM) proportion

A previously described differential staining technique [14] was used to estimate the total cell number (TCN) and the proportion of inner cell mass (ICM) of the blastocysts.

Day seven blastocysts were incubated in 10 $\mathrm{mg} / \mathrm{mL}$ of propidium iodide and $1 \mathrm{mg} / \mathrm{mL}$ of Triton $\mathrm{X}-100$, diluted in Dulbecco's phosphate-buffered saline (D-PBS), for $40 \mathrm{~s}$ at room temperature, being immediately fixed with absolute ethanol added of 15 $\mathrm{mg} / \mathrm{mL}$ of bisbenzimide (Hoecsht 33342) for additional $7 \mathrm{~min}$. Fixed embryos were placed into a $5 \mu \mathrm{L}$ droplet of glycerol, between slide and coverslip, and evaluated through epi-fluorescent inverted microscope ${ }^{2}$. The TCN and the number of cells in the trophectoderm were assessed, being the ICM proportion determined by the difference between these two estimates.

\section{Statistical Analysis}

Data were analyzed using the JMP software version 5, $2002(\mathrm{SAS})^{4}$. Cleavage and blastocyst rates from both experiments were analyzed through the Chi- 
Square test. The rates of oocyte maturation, penetration and fragmentation, as well as blastocyst TCN and proportion of ICM were analyzed by ANOVA (normalized by the arc sin of the square root of the data), and Tukey test. The significance level was always of $5 \%$.

\section{RESULTS}

Maturation and fertilization patterns after immature oocyte vitrification in a base solution partially of totally composed of FF

The maturation rate of oocytes vitrified in $\mathrm{TH}$ (66.7\%) was significantly lower than the Fresh Control (86.8\%). However, when oocytes were vitrified in TH:FF $(75.8 \%)$ or in FF $(78.0 \%)$, they no longer showed statistical differences with other groups, nor between them (Table 1). Fertilization rates were similar for both Fresh Control (94.3\%) and FF-Vitri (92.7\%), both significantly higher than TH-Vitri (75.6\%), being the TH:FF-Vitri $(83.5 \%)$ similar to all other treatments. All the vitrified groups showed more polyspermic and fragmented zygotes $(P \leq 0.05)$ than the Fresh Control (Table 1).
Assessment of embryo development rates and quality after immature oocyte vitrification in a base solution partially of totally composed of FF

The experimental groups that had FF in its base solution showed lower $(P \leq 0.05)$ cleavage rates (TH:FF 51.9\% and FF 45.9\%) than the Fresh Control (66.0\%), but the cleavage rate from TH-Vitri $(55.4 \%)$ was similar to all other groups (Table 2). Blastocyst and morula + blastocyst rates at day 7 were lower in all vitrified groups, if compared to the Fresh Control $(P \leq 0.05)$. Conversely, if estimated based on cleaved, morula + blastocyst rates were similar $(P>0.05)$ for the Fresh Control (43.9\%) and FF-Vitri (31.1\%). The average TCN of blastocysts did not differ $(P>$ 0.05) among the groups Fresh Control (59.8), THVitri (54.6), TH:FF-Vitri (59.7), and FF-Vitri (67.2). Despite being a cryopreserved group, the blastocysts from FF-Vitri $(20.5 \%)$, had similar $(P>0.05)$ ICM proportion (Table 2) as the Fresh Control (24.9\%), in contrast with the lower proportion of their other vitrified counterparts (TH-Vitri $18.1 \%$ and TH:FFVitri $18.0 \%$ ).

Table 1. Evaluation of nuclear status of IVF zygotes derived from fresh or vitrified immature bovine oocytes.

\begin{tabular}{ccccc}
\hline Group & Maturated oocytes & Fertilyzed oocytes & Polyspermic zygotes & Fragmented zygotes \\
& $\mathrm{n}(\%)$ & $\mathrm{n}(\%)$ & $\mathrm{n}(\%)$ & $0)$ \\
\hline Fresh Control & $105 / 121(86.8)^{\mathrm{a}}$ & $99 / 105(94.3)^{\mathrm{a}}$ & $0 / 99(0.0)^{\mathrm{b}}$ & $0 / 99(0.0)^{\mathrm{b}}$ \\
TH-Vitri & $86 / 129(66.7)^{\mathrm{b}}$ & $65 / 86(75.6)^{\mathrm{b}}$ & $7 / 65(10.8)^{\mathrm{a}}$ & $6 / 65(9.2)^{\mathrm{a}}$ \\
TH:FFVitri & $91 / 120(75.8)^{\mathrm{ab}}$ & $76 / 91(83.5)^{\mathrm{ab}}$ & $7 / 76(9.2)^{\mathrm{a}}$ & $7 / 76(9.2)^{\mathrm{a}}$ \\
FF-Vitri & $96 / 123(78.0)^{\mathrm{ab}}$ & $89 / 96(92.7)^{\mathrm{a}}$ & $7 / 89(7.9)^{\mathrm{a}}$ & $7 / 89(7.9)^{\mathrm{a}}$ \\
\hline
\end{tabular}

${ }^{\mathrm{ab} D i s t i n c t ~ l e t t e r s ~ i n ~ t h e ~ s a m e ~ c o l u m n ~ s h o w n ~ d i f f e r e n c e ~}(P \leq 0.05)$. TH-Vitri - Vitrified oocytes in TCM-199 HEPES + 20\% estrus mare serum as base solution. TH:FF-Vitri - Vitrified oocytes in mix (50/50\%) of TCM-199 HEPES + 20\% estrus mare serum and follicular fluid as base solution. FF-Vitri - Vitrified oocytes in follicular fluid as base solution.

Table 2. Evaluation of embryo development rates, average total cell number and inner cell mass (ICM) \pm SE proportion of IVF blastocysts derived from fresh or vitrified immature bovine oocytes.

\begin{tabular}{cccccccc}
\hline & & & & \multicolumn{2}{c}{ Morula + blastocyst } & \multicolumn{2}{c}{ Cells in blastocysts } \\
\cline { 5 - 8 } Group & $\begin{array}{c}\text { Cultured } \\
\mathrm{n}\end{array}$ & $\begin{array}{c}\text { Cleaved } \\
\mathrm{n}(\%)\end{array}$ & $\begin{array}{c}\text { Blastocyst } \\
\mathrm{n}(\%)\end{array}$ & $\begin{array}{c}\text { From culture } \\
\mathrm{n}(\%)\end{array}$ & $\begin{array}{c}\text { From cleaved } \\
(\%)\end{array}$ & $\begin{array}{c}\text { Total cell number } \\
\text { Cells in ICM } \\
(\%)\end{array}$ \\
\hline Fresh Control & 100 & $66(66.0)^{\mathrm{a}}$ & $23(23.0)^{\mathrm{a}}$ & $29(29.0)^{\mathrm{a}}$ & $43.9^{\mathrm{a}}$ & $59.8 \pm 4.7$ & $24.9 \pm 1.9^{\mathrm{a}}$ \\
TH-Vitri & 92 & $51(55.4)^{\mathrm{ab}}$ & $5(5.4)^{\mathrm{b}}$ & $8(8.7)^{\mathrm{b}}$ & $15.7^{\mathrm{b}}$ & $54.6 \pm 5.3$ & $18.1 \pm 2.1^{\mathrm{b}}$ \\
TH:FFVitri & 104 & $54(51.9)^{\mathrm{b}}$ & $4(3.8)^{\mathrm{b}}$ & $11(10.6)^{\mathrm{b}}$ & $20.4^{\mathrm{b}}$ & $59.7 \pm 2.4$ & $18.0 \pm 1.0^{\mathrm{b}}$ \\
FF-Vitri & 98 & $45(45.9)^{\mathrm{b}}$ & $4(4.1)^{\mathrm{b}}$ & $14(14.3)^{\mathrm{b}}$ & $31.1^{\mathrm{ab}}$ & $67.2 \pm 5.3$ & $20.5 \pm 2.1^{\mathrm{ab}}$ \\
\hline
\end{tabular}

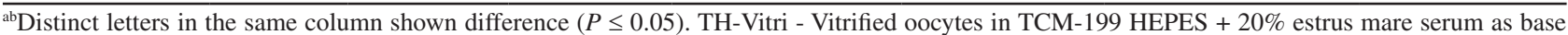
solution. TH:FF-Vitri - Vitrified oocytes in mix (50/50\%) of TCM-199 HEPES + 20\% estrus mare serum and follicular fluid as base solution. FF-Vitri - Vitrified oocytes in follicular fluid as base solution. 
Embryo development rates and quality after IVF or ICSI of vitrified/rewarmed immature oocytes

The cleavage rate for Fresh-IVF (65.6\%) was higher $(P \leq 0.05)$ than for all the other groups (FreshICSI $50.0 \%$, Vitri-IVF $48.3 \%$ and Vitri-ICSI $46.9 \%)$ [Table 3]. Blastocyst rates were higher $(P \leq 0.05)$ for fresh-IVF (26.4\%) and fresh-ICSI (28.9\%) than for Vitri-IVF (4.9\%) and Vitri-ICSI (11.9\%), being VitriICSI also higher than Vitri-IVF (Table 3). When morula + blastocyst rates were calculated with basis on cleaved, this rate was similar between Fresh-IVF (43.9\%) and
Fresh-ICSI (57.8\%), being Fresh-IVF and Vitri-ICSI $(37.3 \%)$ similar between them, but lower than Fresh-ICSI. The lowest rate $(P \leq 0.05)$ in comparison to all other groups (Table 3) was presented by Vitri-IVF (18.8\%).

The mean TCN, as well as the ICM proportion of blastocysts from all groups did not differ $(P>0.05)$ amongst them, being the TCN respectively 58.5, 69.0, 81.7 and 73.5 for Fresh-IVF, Fresh-ICSI, Vitri-IVF and Vitri-ICSI. The ICM proportion was respectively of 22.3, 21.0, 26.9 and 21.6 for Fresh-IVF, Fresh-ICSI, Vitri-IVF and Vitri-ICSI.

Table 3. Evaluation of embryo development rates, average total cell number and inner cell mass (ICM) \pm SE proportion of IVF or ICSI blastocysts derived from fresh or vitrified immature bovine oocytes.

\begin{tabular}{|c|c|c|c|c|c|c|c|}
\hline \multirow[b]{2}{*}{ Group } & \multirow{2}{*}{$\begin{array}{c}\text { Cultured } \\
\mathrm{n}\end{array}$} & \multirow{2}{*}{$\begin{array}{l}\text { Cleaved } \\
\mathrm{n}(\%)\end{array}$} & \multirow{2}{*}{$\begin{array}{c}\text { Blastocyt } \\
\mathrm{n}(\%)\end{array}$} & \multicolumn{2}{|c|}{ Morula + blastocyst } & \multicolumn{2}{|c|}{ Cells in blastocysts } \\
\hline & & & & $\begin{array}{c}\text { From cultured } \\
\mathrm{n}(\%)\end{array}$ & $\begin{array}{l}\text { From cleaved } \\
\qquad(\%)\end{array}$ & $\begin{array}{l}\text { Total cell } \\
\text { number }\end{array}$ & $\begin{array}{c}\text { cells in ICM } \\
(\%)\end{array}$ \\
\hline Fresh-IVF & 125 & $82(65.6)^{\mathrm{a}}$ & $33(26.4)^{\mathrm{a}}$ & $36(28.8)^{\mathrm{a}}$ & $43.9^{\mathrm{ab}}$ & $58.5 \pm 5.4$ & $22.3 \pm 1.2$ \\
\hline Fresh-ICSI & 128 & $64(50.0)^{\mathrm{b}}$ & $37(28.9)^{\mathrm{a}}$ & $37(28.9)^{\mathrm{a}}$ & $57.8^{\mathrm{a}}$ & $69.0 \pm 4.8$ & $21.0 \pm 1.1$ \\
\hline Vitri-IVF & 143 & $69(48.3)^{b}$ & $7(4.9)^{\mathrm{c}}$ & $13(9.1)^{\mathrm{c}}$ & $18.8^{\mathrm{c}}$ & $81.7 \pm 11.3$ & $26.9 \pm 2.5$ \\
\hline Vitri-ICSI & 160 & $75(46.9)^{\mathrm{b}}$ & $19(11.9)^{\mathrm{b}}$ & $28(17.5)^{b}$ & $37.3^{\mathrm{b}}$ & $73.5 \pm 6.8$ & $21.6 \pm 1.5$ \\
\hline
\end{tabular}

\section{DISCUSSION}

Despite the damage caused by cryopreservation, the maturation and fertilization rates after vitrification of GV oocytes in total or partial FF based solution were similar to the Fresh Control (Table 1), evidencing its beneficial effect in the course of vitrification. It is possible that substances present in the FF give them some protection during cryopreservation. This corroborates with previous findings [1], that small follicles have best ability to inhibit the germinal vesicle breakdown.

Reports that oocytes cryopreserved in TH show rigidity in the zona pellucida [20] are a possible link to an impaired spermatozoa penetration [2,3,5]. Our findings agree with that, since the fertilization rate of TH-Vitri was lower than the FF-Vitri (Table 1). Oocytes vitrified in total FF solution yielded a fertilization rate similar to the Fresh Control. The early releasing of cortical granules induced by freezing and thawing is another possible reason to failure in sperm penetration [7]. Indeed, it should be investigated whether FF is responsible for triggering some pathway to prevent this early release of cortical granules, when applied during the course of vitrification.

Intriguingly, the cleavage rates for both treatments that vitrified using FF had the cleavage rate lower than the Fresh Control, unlike the TH-Vitri (Table 2). This needs further investigation in order to pinpoint the reasons why this pattern was observed in the cleavage rates. Still regarding the vitrified/rewarmed oocytes, the blastocysts from the FF-Vitri were the only ones showing the ICM percentage similar to the Fresh Control (Table 2), evidencing that embryos derived from oocytes vitrified in FF had an improved quality. Previous studies have reported that FF contains antioxidants such as catalase [6], which may play a positive role in the defense of oocytes during vitrification.

When vitrified-warmed oocytes were fertilized by ICSI, the blastocyst production was increased (Table 3). This increased embryo production, as previously found in humans [18], evidences that ICSI is an alternative to overcome the zona pellucida barrier, as the sperm cell is inserted directly into the ooplasm. Our data also show increased results of Vitri-ICSI. Conversely, previous findings in the bovine [17] did 
not find any increment in embryo development after MII oocyte vitrification and ICSI, though under their conditions the cleavage rates increased. This study shows that: the composition of the base solution for oocyte vitrification can improve the embryo production outcome, and ICSI improves the embryo production for previously vitrified oocytes. The roles played by the FF during the course of oocyte vitrification should be further investigated. Ultimately, the post-implantation efficiency of ICSI in post-vitrified oocytes should be evaluated, after embryo transfer.

\section{CONCLUSIONS}

Vitrification of GV stage oocytes in FF based solution has a positive effect in the maturation, fertil- ization and embryo development. Vitrification of GV oocytes in a solution totally based in FF improves further embryo quality. In the comparison between ICSI and IVF for the fertilization of oocytes vitrified in FF, ICSI provides the best embryo development rates.

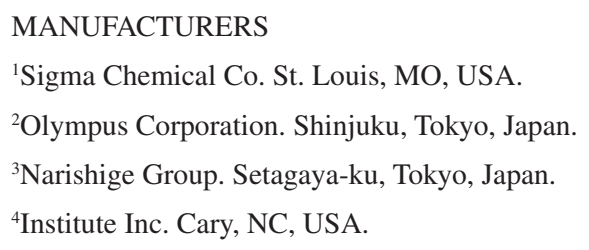

Acknowledgements. The authors wish to thank CAPES and CNPQ for the financial support.

Declaration of interest. The authors declare that they have no conflict of interest that is directly or indirectly related to this research.

\section{REFERENCES}

1 Ayoub M.A. \& Hunter A.G. 1993. Inhibitory Effect of Bovine Follicular Fluid on In Vitro Maturation of Bovine Oocytes. Journal of Dairy Science. 76(1): 95-100.

2 Carroll J., Depypere H. \& Matthews C.D. 1990. Freeze-thaw-induced changes of the zona pellucida explains decreased rates of fertilization in frozen-thawed mouse oocytes. Journal of Reproduction and Fertility. 90(2): 547-553.

3 Carroll J., Warnes G.M. \& Matthews C.D. 1989. Increase in digyny explains polyploidy after in vitro fertilization of frozen-thawed mouse oocytes. Journal of Reproduction and Fertility. 85(2): 489-494.

4 De Loos F., Van Vliet C., Van Maurik P. \& Kruip T.A. 1989. Morphology of immature bovine oocytes. Gamete Research. 24(2): 197-204.

5 Fuku E., Kojima T., Shioya Y., Marcus G.J. \& Downey B.R. 1992. In Vitro Fertilization and Development of FrozenThawed Bovine Oocytes. Cryobiology. 29(4): 485-492.

6 Gupta S., Choi A., Yu H.Y., Czerniak S.M., Holick E.A., Paolella L.J., Agarwal A. \& Combelles C.M.H. 2011. Fluctuations in total antioxidant capacity, catalase activity and hydrogen peroxide levels of follicular fluid during bovine folliculogenesis. Reproduction, Fertility and Development. 23(5): 673-680.

7 Hochi S., Kanamori A., Kimura K. \& Hanada A. 1997. In vitro fertilizing ability of bovine oocytes frozen-thawed at immature, maturing, and mature stages. Journal of Mammalian Ova Research. 14(1): 61-65.

8 Holm P., Booth P.J., Schmidt M.H., Greve T. \& Callesen H. 1999. High bovine blastocyst development in a static in vitro production system using SOFaa medium supplemented with sodium citrate and Myoinositol with or without serum-proteins. Theriogenology. 52(4): 683-700.

9 Kalab P., Kopf G.S. \& Schultz R.M. 1991. Modifications of the mouse zona pellucida during oocyte maturation and egg activation: effects of newborn calf serum and fetuin. Biology of reproduction. 45(5): 783-787.

10 Kalab P., Schultz R.M. \& Kopf G.S. 1993. Modifications of the mouse zona pellucida during oocyte maturation: inhibitory effects of follicular fluid, fetuin, and a2HS-glycoprotein. Biology of Reproduction. 49(3): 561-567.

11 Le Gal F. 1996. In vitro maturation and fertilization of goat oocytes frozen at the germinal vesicle stage. Theriogenology. 45(6): 1177-1185.

12 Machado G.M., Carvalho J.O., Siqueira Filho E., Caixeta E.S., Franco M.M., Rumpf R. \& Dode M.A.N. 2009. Effect of Percoll volume, duration and force of centrifugation, on in vitro production and sex ratio of bovine embryos. Theriogenology. 71(8): 1289-1297.

13 Maddox-Hyttel P., Svarcova O. \& Laurincik J. 2007. Ribosomal RNA and nucleolar proteins from the oocyte are to some degree used for embryonic nucleolar formation in cattle and pig. Theriogenology. 68(Suppl 1): s63-s70.

14 Mezzalira J.C., Ohlweiler L.U., Gerger R.P.C., Casali R., Vieira F.K., Ambrósio C.E., Miglino M.A., Rodrigues J.L., Mezzalira A. \& Bertolini M. 2011. Production of bovine hand-made cloned embryos by zygote-oocyte cytoplasmic Hemi-complementation. Cellular reprogramming. 13(1): 65-76. 
15 Ohlweiler L.U., Brum D.S., Leivas F.G., Moyses A.B., Ramos R.S., Klein N., Mezzalira J.C. \& MezzaliraA. 2013. Intracytoplasmic sperm injection improves in vitro embryo production from poor quality bovine oocytes. Theriogenology. 79(5): 778-783.

16 Parrish J.J., Susko-Parrish J.L., Leibfried-Rutledge M.L., Cristser E.S, Eyestone W.H. \& First N.L. 1986. Bovine in vitro fertilization with frozen-thawed semen. Theriogenology. 25(4): 592-600.

17 Rho G.J., Lee S.L., Kim Y.L., Yeo H.J., Ock S.A., Balasubramanian S. \& Choe S.Y. 2004. Intracytoplasmic sperm injection of frozen-thawed bovine oocytes and subsequent embryo development. Molecular Reproduction and Development. 68(4): 449-455.

18 Rienzi L., Romano S., Albricci L., Maggiulli R., Capalbo A., Baroni E., Colamaria S., Sapienza F. \& Ubaldi F. 2010. Embryo development of fresh 'versus' vitrified metaphase II oocytes after ICSI: a prospective randomized sibling-oocyte study. Human Reproduction. 25(1): 66-73.

19 Rusciano G., De Canditiis C., Zito G., Rubessa M., Roca M.S., Carotenuto R., Sasso A. \& Gasparrini B. 2017. Raman-microscopy investigation of vitrification-induced structural damages in mature bovine oocytes. PLoS One. 12(5): e0177677.

20 Xu K.P. \& Greve T. 1988. A detailed analysis of early events during in-vitro fertilization of bovine follicular oocytes. Journal of Reproduction and Fertility. 82(1): 127-134.

21 Yamada M. \& Isaji Y. 2011. Structural and functional changes linked to, and factors promoting, cytoplasmic maturation in mammalian oocytes. Reproduction in Medicine Biology. 10(2): 69-79. 